\title{
MANAGEMENT OF THIRD AND FOURTH DEGREE PERINEAL TEARS
}

\author{
Joseph Mechery, MBBS, MD; David Burch, MRCOG
}

\section{INTRODUCTION}

It is estimated that $85 \%$ of women will sustain some perineal trauma at delivery and $60 \%$ will require repair. This trauma can cause significant physical and psychological morbidity.

Severe perineal tears occur in approximately $05 \%-0.7 \%$ of vaginal deliveries. Severe tears involving the anal sphincters (grade III tear) and/or rectal mucosa (grade IV tear) have been identified in $0.6 \%-9 \%$ of vaginal deliveries where mediolateral episiotomy is performed ${ }^{(1)}$. The introduction of endoanal ultrasound has identified a much larger proportion of women (up to $36 \%$ ) who sustain occult damage. Standard classification subdivides third degree tears into three subtypes depending on the severity of injury.

\section{PRINCIPLES OF REPAIR OF THIRD AND FOURTH DEGREE PERINEAL TEAR ${ }^{(2,3)}$}

The repair should be performed only by a doctor experienced in the repair of anal sphincter injury. Repair should be conducted in an operating theatre with good lighting, appropriate equipment and under aseptic conditions. General or regional anaesthesia is an important prerequisite and full extent of injury evaluated prior to repair. When a fourth degree tear is present, the rectal mucosa is repaired by interrupted 3-0 polyglactin sutures (Vicryl) and the knots tied in the rectal lumen. It is recommended that sphincter muscles be repaired with 3-0 polydioxalone dyed sutures, such as PDS. Internal and external sphincters should be repaired separately. For internal sphincter repair, an end-to-end repair is performed as overlapping can be technically difficult. Endto-end repair with figure of eight sutures is the most commonly used technique for external anal sphincter repair. Muscles of the perineal body are sutured with interrupted 2-0 Vicryl sutures after closing the vaginal epithelium with a continuous Vicryl 3-0 sutures. The perineal skin is approximated with 3-0 Vicryl subcuticular sutures. Rectovaginal examination is performed to ensure complete repair and antibiotics given for a week. A Foleys catheter is inserted to avoid urinary retention due to perineal pain and regional anaesthesia. Clear documentation is essential. Stool softeners and bulking agents should be prescribed for at least two weeks to avoid damage to the repair.

\section{FOLLOW-UP AND COUNSELLING}

All women who sustain third or fourth degree perineal repair should be assessed by a senior obstetrician six to eight weeks after delivery. A careful history and examination should be performed to check healing, scar tenderness and sphincter tone $\mathrm{e}^{(4)}$. Symptomatic women should be referred to a specialist centre for further investigations (endoanal ultrasound, anal manometry) and management. Asymptomatic women who do not have compromised anal sphincter function can be advised to undergo vaginal delivery. Women who have had a previous successful secondary sphincter repair for faecal incontinence and those who sustained third or fourth degree tear with subsequent severe incontinence should be delivered by Caesarean section ${ }^{(5)}$. Severe incontinence following a third or fourth degree repair should be offered secondary sphincter repair by a colorectal surgeon ${ }^{(6)}$.

\section{AUDIT ON MANAGEMENT OF THIRD AND FOURTH DEGREE PERINEAL TEAR FOLLOWING VAGINAL DELIVERY}

We undertook a re-audit to determine whether the recommendations of the previous audit were implemented, to identify rate of tears and to confirm we follow the management plan recommended by the Royal College of Obstetricians and Gynaecologists.

We retrospectively analysed all cases of third and fourth degree tears managed at the Royal Lancaster Infirmary from 1st January to 31st December 2004.

There were fourteen cases of third degree and four cases of fourth degree perineal tears. The overall rate was $1.1 \%$. We found that $14(88 \%)$ were repaired in theatre under general anaesthesia or regional block. Ninety-four per cent of cases were repaired by registrars. All had laxatives and antibiotics and 94\% had six-week follow-up (an improvement from the previous audit). At follow-up, only one was symptomatic. There was poor documentation with respect to degree of sphincter damage and counselling regarding subsequent delivery.

We recommended:

- continued training in classification and repair for all senior resident doctors

- revision of the existing protocol and clear instructions with regards to the type of suture material used.

- attachment of pro-forma in patients' case notes with boxes for subtype classification and counselling regarding subsequent delivery and to re-audit to confirm these are being implemented

\section{REFERENCES}

1. Royal College of Obstetricians and Gynaecologists. Management of third and fourth degree perineal tears following vaginal delivery. Guideline No. 29. London: RCOG press; 2001

2. Sultan AH, Kahn MA. Perineal and primary anal sphincter repairs. IN Cardozo L, Staskin D, editors. Textbook of 
Female Urology and Urogynaecology. London: ISIS Medical media; 2001.p. 627-42

3. Sultan AN. Third degree tear repair. IN MacLean AB, Cardozo L, editors. Incontinence in women. London: RCOG press; 2002. p. 379-90

4. Sultan AH, Abulafi MA. Anal incontinence - the role of the obstetrician and gynaecologist. IN Sturdee D, Ola`h K, Keane D, editors. The Year Book of Obstetrics and Gynaecology. Volume 9. London: RCOG Press; 2001. p.170-87
5. Fynes M, Donnelly V, Behan M. Effect of second vaginal delivery on anorectal physiology and faecal incontinence: a prospective study. Lancet 1999;354:983-6

6. Thakar R, Sultan AH. Management of obstetric anal sphincter injury. The Obstetrician and Gynaecologist 2003;5:72-8

\section{RECYCLING THE BNF}

The British National Formulary (BNF) is published twice a year and issued free to NHS doctors, dentists, pharmacies and hospitals. It is always there, like air and water, and like them invaluable but unregarded. In other parts of the world where textbooks are never seen the BNF is a precious item, for its drug information and condensed articles on the pharmacology of each drug group.

Through their Pharmaid scheme, the Commonwealth Pharmaceutical Association collects old copies in the United Kingdom and distributes them in many developing countries. The books are collected by the van drivers of AAH Pharmaceuticals, and shipped to Commonwealth countries by BookAid International. This scheme is based on pharmacies, but AAH are willing to collect reasonable numbers from other collecting points.

The scheme has been running for many years, but its pharmacy base means that relatively few BNFs get recycled; only 5000 last year. If hospitals and practices were involved, the numbers re-used could be much greater.

As we have just had a new issue, No. 49, please may I have your old copies? Not very old, Nos. 47 and 48 only, but as soon as possible, please, before they go in the bin!

John Davies

Department of Anaesthesia, Royal Lancaster Infirmary 